\title{
Gradient-Index Polymer Optical Fiber Preparation through a Co-Extrusion Process
}

\author{
Bo-Tau Liu, Meng-Ying HsieH, Wen-Chang CHEN, and Jyh-Ping Hsu ${ }^{\dagger}$ \\ Department of Chemical Engineering, National Taiwan University, \\ Taipei, Taiwan 10617, Republic of China
}

(Received July 15, 1998)

\begin{abstract}
The preparation of gradient index polymer optical fiber through a co-extrusion process is analyzed theoretically. The effects of the essential parameters of the system under consideration on the radial distribution of the refractive index $(R I)$ of the optical fiber are examined through numerical simulation. These include the ratio (volume of inner layer/volume of outer layer), the diffusivity and the initial concentration of monomer, the length of diffusion zone, and the mass transfer coefficient of monomer at the outer boundary of the outer layer. We show that these parameters can play a significant role in the design of a co-extrusion process. If they are chosen appropriately, about $80 \%$ of an optical fiber can have an approximate parabolic $R I$ distribution in its radial direction.
\end{abstract}

KEY WORDS Co-Extrusion Process / Optical Fiber Preparation/Mathematical Modeling/

Gradient-index (GI) polymer is one of the most popular materials for optical fiber communication and imaging. ${ }^{1-5}$ Reported results for potential applications of GI polymer optical fiber are ample in the literature. These include, for example, a high bandwidth GI polymer optical fiber, ${ }^{6}$ GI polymer optical fiber amplifier with a high gain in the visible region, ${ }^{7}$ and GI polymer lens used in fax machines. ${ }^{8,9}$

It is known that the bandwidth can be maximized if the radial distribution of the refractive index $(R I)$ of an optical fiber is parabolic. ${ }^{10}$ Several efforts have been made to achieve this idealized condition. Perry and Witcher, ${ }^{11}$ for instance, proposed a multiple layer co-extrusion process, which is capable of yielding a near parabolic RI distribution. Similar idea was also adopted by other researchers. ${ }^{9,12-14}$ Yamamoto et al. ${ }^{8}$ Mishina et al. ${ }^{15,16}$ and Mishina et al., ${ }^{17}$ were able to design an extrusion process, which comprises an internal diffusion and surface evaporation operation. Another possible approach is the closed co-extrusion process developed by Ho et al. ${ }^{12}$ Chen et al.,${ }^{13}$ and Chen, et al. ${ }^{14}$ It was shown experimentally that this process has the advantage of high reproducibility and high production rate. The mathematical analysis on the closed co-extrusion process was presented recently by Liu et al. ${ }^{18}$ in which the effects of the nature of the reactant mixtures and the operating conditions such as different volume ratios of (inner layer/outer layer), extrusion velocity, and diffusion length on the radial distribution of $R I$ were discussed. It was shown that the radial distribution of $R I$ can be affected appreciably by these factors. However, achieving an exact parabolic $R I$ distribution for the entire cross section of an optical fiber is impossible. This is because that the outer boundary of the co-extrusion apparatus is impermeable to the diffusing monomers, and the radial distribution of $R I$ becomes flat near the outer boundary of the extruded fiber.

In the present study, the analysis of Liu et al. ${ }^{18}$ is extended to the case where a more general condition on the outer boundary of the co-extrusion apparatus is assumed. This not only provides a more flexible design for the apparatus, but also improves the $R I$ profile in an extruded fiber.

\section{THEORY}

The system under consideration is the same as that of Liu et al. ${ }^{18}$ except that the outer boundary of the diffusion zone is permeable to monomers. A schematic representation of the diffusion zone is illustrated in Figure 1, where $R_{\mathrm{f}}$ denotes the radius of the inner layer of the diffusion zone, $R$ and $L$ are the radius and the length of the diffusion zone, respectively, $r$ is the radial distance, $z$ denotes the distance from the inlet of the diffusion zone,

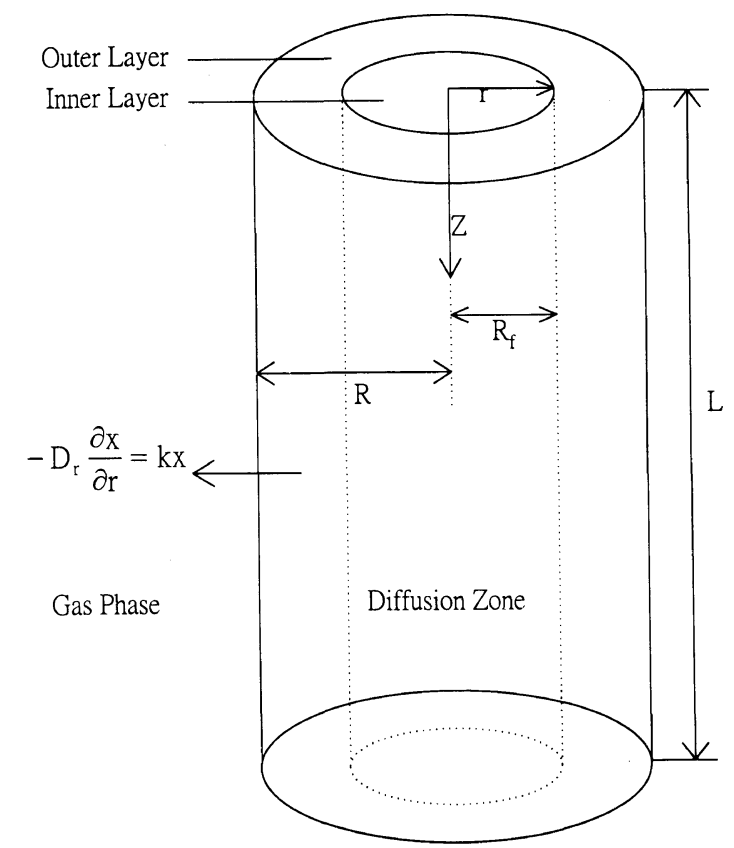

Figure 1. Coordinates adopted in the mathematical modeling, $r$ and $z$ are the radial and axial distances, respectively, $L$ is the length of diffusion zone, $R_{\mathrm{f}}$ and $R$ are, respectively, the radii of the inner and outer layers of the diffusion zone.

\footnotetext{
${ }^{\dagger}$ To whom correspondence should be addressed (Fax: +886-2-23623040, e-mail: t8504009@ ccms.ntu.edu.tw).
} 
and the region $r>R$ represents the gas phase. The feed to the inner layer contains polymer PA, its monomer A, and monomer $\mathrm{B}$, and that to the outer layer comprises polymer PA and monomer $\mathrm{A}$. In passing through the diffusion zone, monomer $\mathrm{B}$ will diffuse toward the outer layer, and since the outer boundary of the outer layer is permeable to monomers, both $\mathrm{A}$ and $\mathrm{B}$ may diffuse to the gas phase. This leads to a radial distribution in the concentrations of monomers A and B. If the filament at the outlet of the diffusion zone is hardened, monomers $\mathrm{A}$ and $\mathrm{B}$ are polymerized to $\mathrm{PA}$ and $\mathrm{PB}$ respectively. Suppose that the $R I$ of $\mathrm{PB}$ is greater than that of $\mathrm{PA}$. For simplicity, we assume that the operation is isothermal, and the diffusivities of $\mathrm{A}$ and $\mathrm{B}$ are constant. Also, we assume that the bulk densities of the inner and the outer layers are constant. Under these conditions, the spatial variation in the mass fraction of monomer (A or B), $x$, at the steady-state operation can be described by

$$
u \frac{\partial x}{\partial z}=D_{\mathrm{r}}\left[\frac{\partial^{2} x}{\partial r^{2}}+\frac{1}{r} \frac{\partial x}{\partial r}\right]+D_{z} \frac{\partial^{2} x}{\partial z^{2}}
$$

Here, $u$ is the extrusion velocity, $t$ denotes the time, and $D_{r}$ and $D_{z}$ are the effective diffusivities of monomer in $r$ and $z$ directions, respectively. We assume that the boundary conditions associated with eq 1 are

$$
\begin{gathered}
x \quad \text { is finite at } r=0 \\
-D_{r} \frac{\partial x}{\partial r}=k x \text { at } r=R
\end{gathered}
$$

where $k$ represents the mass transfer coefficient of monomer at the outer boundary of the outer layer of the diffusion zone. The inlet conditions for monomer B are

$$
\begin{array}{cc}
x=x_{\mathrm{Bi}}, & 0<r<R_{\mathrm{f}} \\
x=0, & R_{\mathrm{f}}<r<R
\end{array}
$$

where $x_{\mathrm{Bi}}$ is the inlet mass fraction of monomer $\mathrm{B}$ in the inner layer. Similarly, the inlet conditions for monomer A are

$$
\begin{array}{ll}
x=x_{\mathrm{Ai}}, & 0<r<R_{\mathrm{f}} \\
x=x_{\mathrm{Ao}}, & R_{\mathrm{f}}<r<R
\end{array}
$$

where $x_{\mathrm{Ai}}$ and $x_{\mathrm{Ao}}$ are respectively the inlet mass fractions of monomer $\mathrm{A}$ in the inner and the outer layers. Suppose that the Peclect number, $\left(u L / D_{z}\right)$, is large, that is, the transport of monomers due to convective motion is much more significant than that due to molecular diffusion. This is usually satisfied for conditions of practical significance. In this case, the last term on the right-hand side of eq 1 is negligible, and we have, after some algebraic manipulations,

$$
\frac{\partial x^{*}}{\partial z^{*}}=\frac{\partial^{2} x^{*}}{\partial r^{* 2}}+\frac{1}{r^{*}} \frac{\partial x^{*}}{\partial r^{*}}
$$

where $x^{*}=x / x_{0}, r^{*}=r / R, x_{0}$ being the mass fraction of monomer at the inlet of the diffusion zone $(z=0)$, and

$$
z^{*}=\frac{z D_{\mathrm{r}}}{u R^{2}}
$$

Note that for monomer $\mathrm{B}, x_{0}=x_{\mathrm{Bi}}$, and for monomer $\mathrm{A}, x_{0}=x_{\mathrm{Ao}}-x_{\mathrm{Ai}} \equiv x_{\mathrm{Ad}}$. The boundary conditions associated with eq 3 become

$$
\begin{aligned}
& x^{*} \text { is finite at } r^{*}=0 \\
& -\frac{\partial x^{*}}{\partial r^{*}}=k^{*} x^{*} \text { at } r^{*}=1
\end{aligned}
$$

where $k^{*}=k R / D_{r}$. For monomer B, $x^{*}=x_{\mathrm{B}}^{*}=x_{\mathrm{B}} / x_{\mathrm{Bi}}$, we have

$$
\begin{array}{ll}
x^{*}=1, & 0<r^{*}<R_{\mathrm{f}}^{*} \\
x^{*}=0, & R_{\mathrm{f}}^{*}<r^{*}<1
\end{array}
$$

Similarly, for monomer A, $x^{*}=x_{\mathrm{A}}^{*}=x_{\mathrm{A}} / x_{\mathrm{Ad}}$,

$$
\begin{gathered}
x^{*}=x_{\mathrm{Ai}} / x_{\mathrm{Ad}} \equiv x_{\mathrm{Ai}}^{*}, \quad 0<r^{*}<R_{\mathrm{f}}^{*} \\
x^{*}=x_{\mathrm{Ao}} / x_{\mathrm{Ad}}, \quad R_{\mathrm{f}}^{*}<r^{*}<1
\end{gathered}
$$

where $R_{\mathrm{f}}^{*}=R_{\mathrm{f}} / R$. Solving eq 3 subject to eq $4 \mathrm{a}-4 \mathrm{~d}$ gives the scaled radial distribution of the mass fraction of monomer $\mathrm{B}$ at the outlet of the diffusion zone $(z=L)$. We have

$$
x_{\mathrm{B}}^{*}=2 R_{\mathrm{f}}^{*} \sum_{m=1}^{\infty} \frac{\lambda_{m} J_{1}\left(\lambda_{m} R_{\mathrm{f}}^{*}\right)}{J_{0}^{2}\left(\lambda_{m}\right)\left(k^{* 2}+\lambda_{m}^{2}\right)} J_{0}\left(\lambda_{m} r^{*}\right) \exp \left(-\lambda_{m}^{2} z_{\mathbf{B}}^{*}\right)
$$

Similarly, solving eq 3 subject to eq $4 \mathrm{a}, 4 \mathrm{~b}, 4 \mathrm{e}$, and $4 \mathrm{f}$ yields the radial distribution of the scaled mass fraction of monomer A at the outlet of the diffusion zone $(z=L)$. We obtain

$$
\begin{aligned}
x_{\mathrm{A}}^{*}= & x_{\mathrm{Ai}}^{*} \\
+ & 2 \sum_{m=1}^{\infty} \frac{k^{*} J_{0}\left(\lambda_{m}\right)-R_{f}^{*} \lambda_{m} J_{1}\left(\lambda_{m} R_{f}^{*}\right)}{J_{0}^{2}\left(\lambda_{m}\right)\left(k^{* 2}+\lambda_{m}^{2}\right)} J_{0}\left(\lambda_{m} r^{*}\right) \\
& \times \exp \left(-\lambda_{m}^{2} z_{\mathrm{B}}^{*}\right)
\end{aligned}
$$

In these expressions, $z_{\mathrm{B}}^{*}=L D_{\mathrm{B}} / u R^{2}, z_{\mathrm{A}}^{*}=L D_{\mathrm{A}} / u R^{2}, J_{0}$ and $J_{1}$ being the Bessel functions of the first kind of orders 0 and 1 respectively, and $\lambda_{m}$ is the positive root of the equation

$$
k^{*} J_{0}\left(\lambda_{m}\right)-\lambda_{m} J_{1}\left(\lambda_{m}\right)=0
$$

\section{Extreme Cases}

Two extreme cases can be recovered directly from the present model: $k^{*}$ is very small $\left(k^{*} \rightarrow 0\right)$, and $k^{*}$ is very large $\left(k^{*} \rightarrow \infty\right)$. The former corresponds to a closed co-extrusion, which was examined by Liu et al. ${ }^{18}$ In this case, eq $5 \mathrm{a}$ and $5 \mathrm{~b}$ become respectively

$$
x_{\mathrm{B}}^{*}=R_{\mathrm{f}}^{* 2}+2 R_{\mathrm{f}}^{*} \sum_{m=1}^{\infty} \frac{J_{1}\left(\lambda_{m} R_{\mathrm{f}}^{*}\right)}{J_{0}^{2}\left(\lambda_{m}\right) \lambda_{m}} J_{0}\left(\lambda_{m} r^{*}\right) \exp \left(-\lambda_{m}^{2} z_{\mathrm{B}}^{*}\right)
$$

and

$$
\begin{aligned}
x_{\mathrm{A}}^{*}= & \left(1-R_{\mathrm{f}}^{* 2}\right) \\
& -2 R_{\mathrm{f}}^{*} \sum_{m=1}^{\infty} \frac{J_{1}\left(\lambda_{m} R_{\mathrm{f}}^{*}\right)}{J_{0}^{2}\left(\lambda_{m}\right) \lambda_{m}} J_{0}\left(\lambda_{m} r^{*}\right) \exp \left(-\lambda_{m}^{2} z_{\mathrm{B}}^{*}\right)+x_{\mathrm{Ai}}^{*}
\end{aligned}
$$

where $\lambda_{m}$ is the positive root of the equation 


$$
J_{1}\left(\lambda_{m}\right)=0
$$

Equations (6a)-(6c) are consistent with the results of Liu et al. ${ }^{18}$

The other extreme, $k^{*} \rightarrow \infty$, implies that a perfect permeability at the outer boundary of the diffusion zone. In this case, eq $5 \mathrm{a}$ and $5 \mathrm{~b}$ reduce to respectively

$$
x_{\mathrm{B}}^{*}=2 R_{f}^{*} \sum_{m=1}^{\infty} \frac{\lambda_{m} J_{1}\left(\lambda_{m} R_{\mathrm{f}}^{*}\right)}{J_{1}^{2}\left(\lambda_{m}\right) \lambda_{m}^{2}} J_{0}\left(\lambda_{m} r^{*}\right) \exp \left(-\lambda_{m}^{2} z_{\mathrm{B}}^{*}\right)
$$

and

$$
\begin{aligned}
x_{\mathrm{A}}^{*}= & x_{\mathrm{A} \mathbf{i}}^{*} \\
+ & 2 \sum_{m=1}^{\infty} \frac{\lambda_{m} J_{1}\left(\lambda_{m}\right)-R_{\mathrm{f}}^{* 2} \lambda_{m} J_{1}\left(\lambda_{m} R_{\mathrm{f}}^{*}\right)}{J_{1}^{2}\left(J_{m}\right) \lambda_{m}^{2}} J_{0}\left(\lambda_{m} r^{*}\right) \\
& \times \exp \left(-\lambda_{m}^{2} z_{\mathrm{B}}^{*}\right)
\end{aligned}
$$

where $\lambda_{m}$ is the positive root of the equation

$$
J_{0}\left(\lambda_{m}\right)=0
$$

After passing the diffusion zone, the filament was hardened, and an optical fiber comprises PA and PB will result. Its $R I, n_{\mathrm{d}}$, can be estimated by the method suggested by Lorentz ${ }^{19}$ and Lorenz. ${ }^{20}$ We have ${ }^{21}$

$$
n_{\mathrm{d}}=\sqrt{\frac{1+2 \phi}{1-\phi}}
$$

The parameter $\phi$ is defined as

$$
\phi=\left(\sum_{i} \frac{n_{\mathrm{d}, i}^{2}-1}{n_{\mathrm{d}, i}^{2}+2} \times \frac{x_{i}}{\rho_{i}}\right) /\left(\sum_{i} \frac{x_{i}}{\rho_{i}}\right)
$$

where $n_{\mathrm{d}, i}$ and $\rho_{i}, i=\mathrm{PA}$ or $\mathrm{PB}$, are the $R I$ and the density of component $i$, respectively.

\section{RESULTS AND DISCUSSION}

Figure 2 shows the variation of $\Delta n=n_{\mathrm{d}}(z=L, r)-n_{\mathrm{d}}$ $(z=L, r=R)$ as a function of $r^{* 2}$ at various ratios $R_{\mathrm{f}} / R$ (=volume of inner layer/volume of outer layer), $R_{\mathrm{f}}^{*}$. This figure reveals that, for $R_{\mathrm{f}}^{*} \leqq 0.7$, and a fixed $r^{* 2}, \Delta n$ increases with $R_{\mathrm{f}}^{*}$. If the ratio $R_{\mathrm{f}}^{*}$ is too small, then the radial variation in $\Delta n$ becomes inappreciable. On the other hand, if $R_{\mathrm{f}}^{*}$ is too large, the distribution of $\Delta n$ near the center of a fiber becomes flat. This is because that if $R_{\mathrm{f}}^{*}$ is small, the amount of monomer $\mathrm{B}$, which has a high $R I$, is low, and therefore the increase in $R I$ due to its presence becomes insignificant. On the other hand, if $R_{\mathrm{f}}^{*}$ is too large, the amount of monomer B is large, the contribution to $R I$ is mainly due to its presence, and $\Delta n$ becomes nearly constant. Note that, if the radial variation of $R I$ is parabolic, then $\Delta n$ is a linear function of $r^{* 2} \cdot{ }^{15}$ Figure 2 suggests that a medium value of $R_{\mathrm{f}}^{*}$ should be adopted.

The variation of $\Delta n$ as a function of $r^{* 2}$ at various $z_{\mathrm{B}}^{*}$ is illustrated in Figure 3; that at various $z_{A}^{*}$ is presented in Figure 4. Figure 3 reveals that the greater the $z_{\mathbf{B}}^{*}$, the closer the distribution of $\Delta n$ to a straight line. Note that, however, the greater the $z_{\mathrm{B}}^{*}$, the smaller the $\Delta n$. Figure 4 suggests that, in general, the distribution of $\Delta n$ for a larger $z_{\mathrm{A}}^{*}$ is closer to a straight line than that for a smaller $z_{\mathrm{A}}^{*}$.

Polym. J., Vol. 31, No. 3, 1999

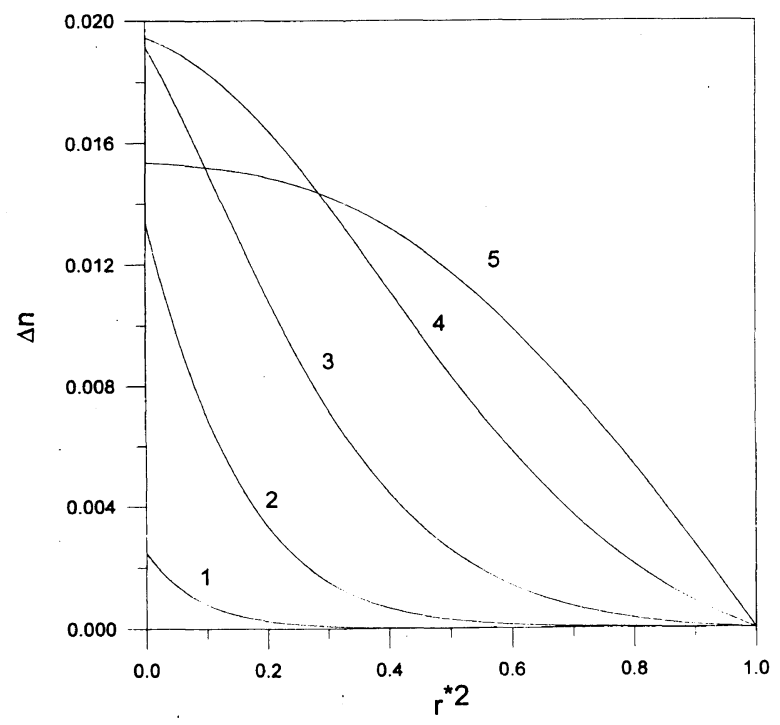

Figure 2. Variation of $\Delta n$ as a function of $r^{* 2}$ at various $R_{\mathrm{f}}^{*}$ for the case $k^{*}=10, z_{\mathrm{A}}^{*}=0.25$, and $z_{\mathrm{B}}^{*}=0.1$. Curve $1: R_{\mathrm{f}}^{*}=0.1,2: R_{\mathrm{f}}^{*}=0.3,3$ : $R_{\mathrm{f}}^{*}=0.5,4: R_{\mathrm{f}}^{*}=0.7,5: R_{\mathrm{f}}^{*}=0.9$. Key: $x_{\mathrm{Ai}}=0.14, x_{\mathrm{Ad}}=0.28, x_{\mathrm{Bi}}=0.28$, $n_{\mathrm{d}, \mathrm{PA}}=1.49, n_{\mathrm{d}, \mathrm{PB}}=1.568$, and the mass fraction of polymer $\mathrm{P}$ is 0.58 in both inner and outer layers.

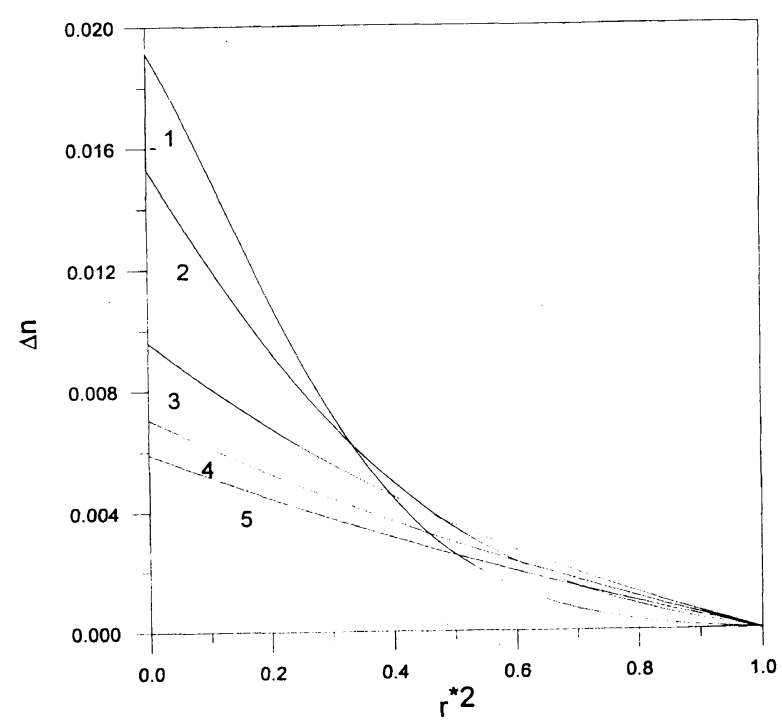

Figure 3. Variation of $\Delta n$ as a function of $r^{* 2}$ at various $z_{\mathrm{B}}^{*}$ for the case $R_{\mathrm{f}}^{*}=0.5, k^{*}=10$, and $z_{\mathrm{A}}^{*}=0.25$. Curve 1: $z_{\mathrm{B}}^{*}=0.02,2: z_{\mathrm{B}}^{*}=0.04$, 3: $z_{\mathbf{B}}^{*}=0.1,4: z_{\mathbf{B}}^{*}=0.16,5: z_{\mathbf{B}}^{*}=0.2$. Key: same as Figure 2 .

Figure 5 shows the variation of $\Delta n$ as a function of $r^{* 2}$ at various combinations of $z_{\mathrm{A}}^{*}$ and $z_{\mathrm{B}}^{*}$. According to Figures 3 and 4 , the larger the value of $z_{\mathrm{A}}^{*}$ (or $z_{\mathrm{B}}^{*}$ ) the closer the distribution of $\Delta n$ to a straight line. Figure 5 reveals that the larger the $z_{\mathrm{A}}^{*}$ and $z_{\mathrm{B}}^{*}$, the closer the distribution of $\Delta n$ to a straight line. Note that, according to eq $3 \mathrm{a}$, a large $z_{\mathrm{A}}^{*}$ (or $z_{\mathrm{B}}^{*}$ ) implies that a large $D_{\mathrm{A}}\left(\right.$ or $D_{\mathrm{B}}$ ), a long $L$, a high $u$, and/or a small $R$. Figure 5 reveals that under these conditions the distribution of $\Delta n$ will approach a limit value, which is close to a straight line.

The variation of $\Delta n$ as a function of $r^{* 2}$ at various inlet concentrations of monomer $\mathbf{B}, x_{\mathrm{Bi}}$, is presented in Figure 6. As can be seen from this figure, for a fixed $r^{* 2}$, $\Delta n$ increases with $x_{\mathbf{B i}}$. This is expected since the $R I$ of monomer $\mathbf{B}$ is greater than that of monomer $\mathbf{A}$. Figure 6 also suggests that the smaller the $x_{\mathrm{Bi}}$, the closer the 


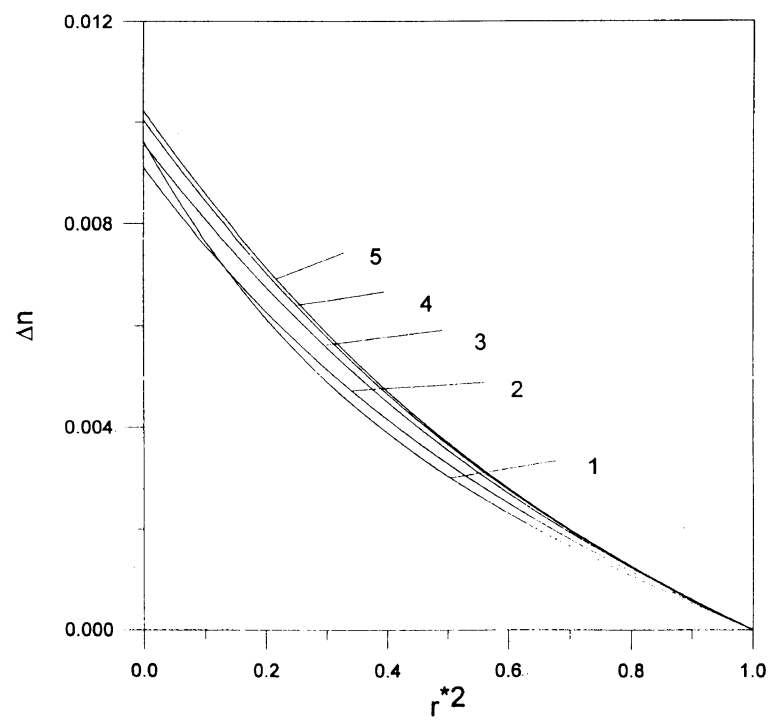

Figure 4. Variation of $\Delta n$ as a function of $r^{* 2}$ at various $z_{\mathrm{A}}^{*}$ for the case $R_{\mathrm{f}}^{*}=0.5, k^{*}=10$, and $z_{\mathrm{B}}^{*}=0.1$. Curve 1: $z_{\mathrm{A}}^{*}=0.05,2: z_{\mathrm{A}}^{*}=0.1,3$ : $z_{\mathrm{A}}^{*}=0.25,4: z_{\mathrm{A}}^{*}=0.4,5: z_{\mathrm{A}}^{*}=0.5$. Key: same as Figure 2 .

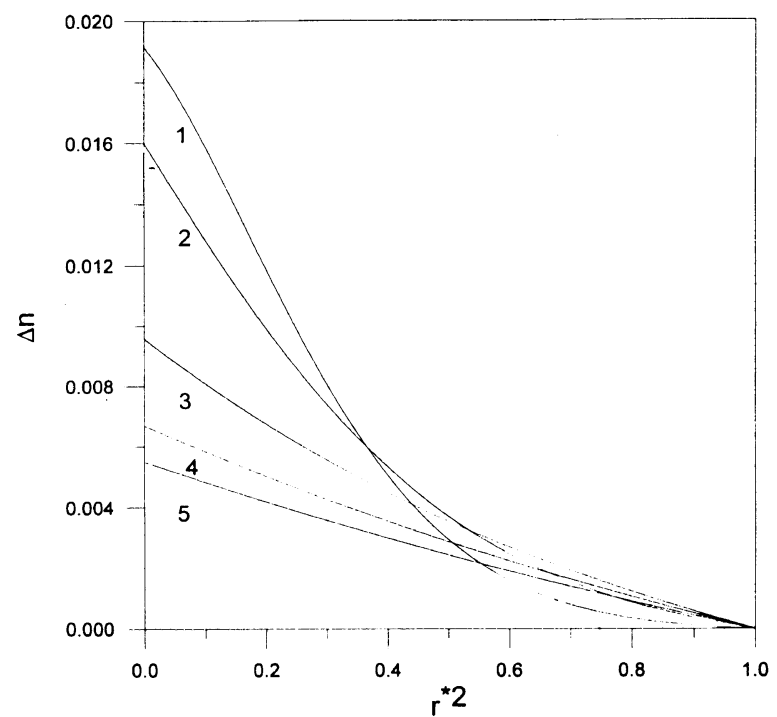

Figure 5. Variation of $\Delta n$ as a function of $r^{* 2}$ at various $z_{\mathrm{A}}^{*}$ and $z_{\mathrm{B}}^{*}$ for the case $k^{*}=10$, and $R_{\mathrm{f}}^{*}=0.5$. Curve $1: z_{\mathrm{A}}^{*}=0.05$ and $z_{\mathrm{B}}^{*}=0.02,2$ : $z_{\mathrm{A}}^{*}=0.1$ and $z_{\mathrm{B}}^{*}=0.04,3: z_{\mathrm{A}}^{*}=0.25$, and $z_{\mathrm{B}}^{*}=0.1,4: z_{\mathrm{A}}^{*}=0.4$, and $z_{\mathbf{B}}^{*}=0.16,5: z_{\mathbf{A}}^{*}=0.5$ and $z_{\mathbf{B}}^{*}=0.2$. Key: same as Figure 2 .

distribution of $\Delta n$ to a straight line. However, if $x_{\mathrm{Bi}}$ is too small, the radial variation of $\Delta n$ may become inappreciable.

Figure 7 shows the variation of $\Delta n$ as a function of $r^{* 2}$ at various scaled mass transfer coefficients at the outer boundary of the outer layer of a fiber, $k^{*}$. This figure reveals that, in general, the larger the $k^{*}$, the more satisfactory the distribution of $\Delta n$. A larger $k^{*}$ implies that the better transfer of monomer through the outer boundary of the outer layer of the diffusion zone to the gas phase. This can be achieved by control appropriately the operating conditions. For instance, if the gas phase flows perpendicularly to the diffusion zone with a Reynolds number about $3000, k^{*}$ can be on the order of $30 .^{22}$

On the basis of Figures 2 through 7, we conclude that the radial distribution of the $R I$ of an optical fiber can

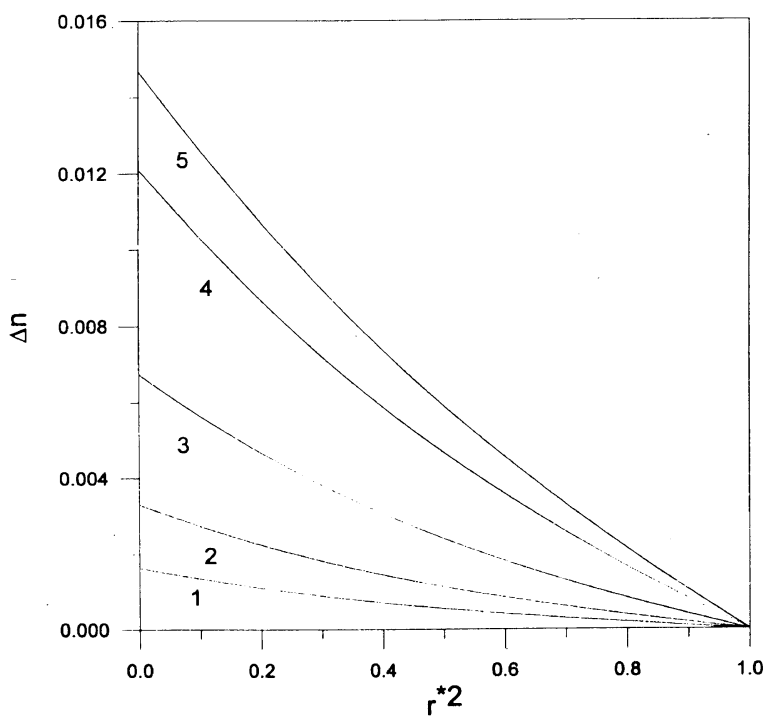

Figure 6. Variation of $\Delta n$ as a function of $r^{* 2}$ at various $x_{\mathrm{Bi}}$ for the case $R_{\mathrm{f}}^{*}=0.5, z_{\mathrm{A}}^{*}=0.25$, and $z_{\mathrm{B}}^{*}=0.1, x_{\mathrm{Ai}}=0.42-x_{\mathrm{Bi}}, x_{\mathrm{Ad}}=0.42-x_{\mathrm{Ai}}$, and the mass fraction of polymer $\mathrm{P}$ is 0.58 for both inner and outer layers. Curve 1: $x_{\mathrm{Bi}}=0.05,2: x_{\mathrm{Bi}}=0.1,3: x_{\mathrm{Bi}}=0.2,4: x_{\mathrm{Bi}}=0.35,5$ : $x_{\mathrm{Bi}}=0.42$. Key: same as Figure 2 .

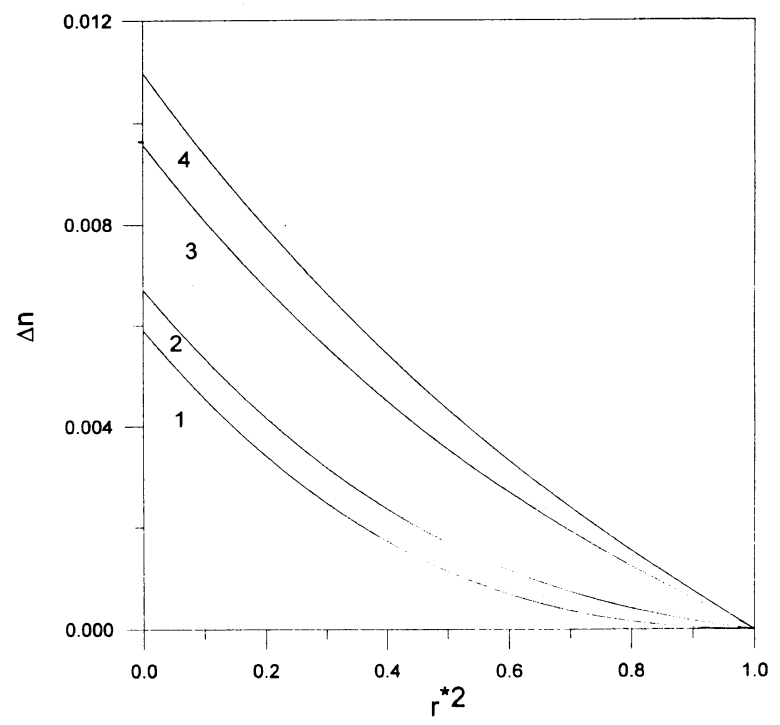

Figure 7. Variation of $\Delta n$ as a function of $r^{* 2}$ at various $k^{*}$ for the case $R_{\mathrm{f}}^{*}=0.5, z_{\mathrm{A}}^{*}=0.25$, and $z_{\mathrm{B}}^{*}=0.1$. Curve 1: $k^{*}=0,2: k^{*}=1,3$ : $k^{*}=10,4: k^{*}=\infty$. Key: same as Figure 2 .

be affected by the ratio (volume of inner layer/volume of outer layer), the diffusivity and the concentration of monomers, the length of diffusion zone, and the mass transfer coefficient of monomer at the outer boundary of the outer layer of a fiber. To achieve the optimum radial distribution for $R I$, these parameters need to be chosen appropriately. Figure 8 shows the $R I$ distribution for the case $R_{\mathrm{f}}^{*}=0.7, \quad z_{\mathrm{A}}^{*}=1, \quad z_{\mathrm{B}}^{*}=0.4, \quad x_{\mathrm{Ai}}=0.07$, $x_{\mathrm{Ao}}=0.42, x_{\mathrm{Bi}}=0.35, x_{\mathrm{P}}=0.58$, and $k^{*} \rightarrow \infty$. As can be seen from this figure, the radial distribution of $R I$ follows roughly a parabolic relationship for about $80 \%$ of the optical fiber. Compared with the design of Liu et al., ${ }^{18}$ in which about $70 \%$ of a fiber can have a parabolic distribution in $R I$, the improvement in the distribution of $R I$ by adopting the present design is about $10 \%$. 


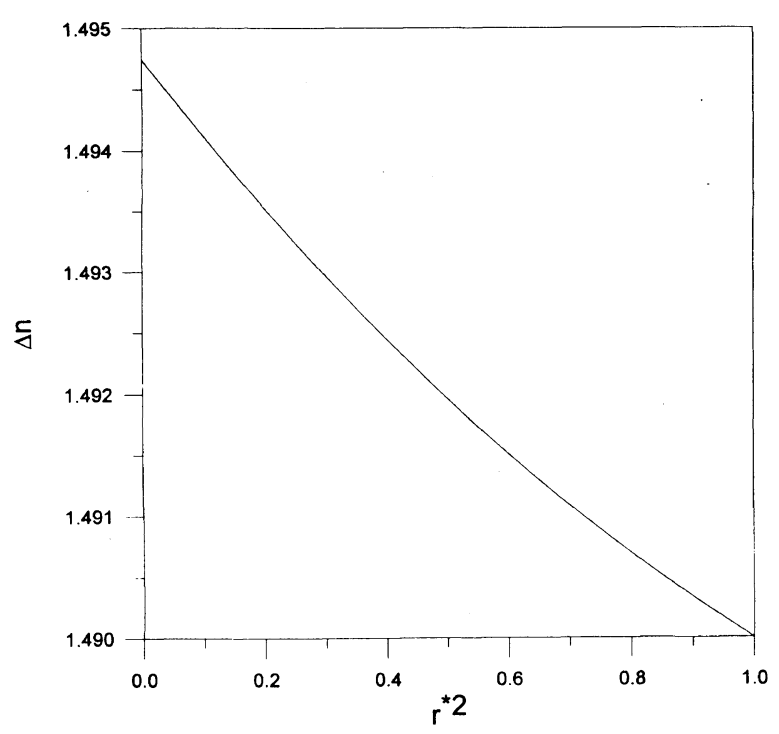

Figure 8. Variation of $\Delta n$ as a function of $r^{* 2}$ at the optimum condition. The values of the parameters are $R_{\mathrm{f}}^{*}=0.7, z_{\mathrm{A}}^{*}=1, z_{\mathrm{B}}^{*}=0.4$, $x_{\mathrm{Bi}}=0.35$, and $k^{*}=\infty$. Key: same as Figure 2 .

Acknowledgment. This work is partially and financially supported by the National Science Council of the Republic of China.

\section{REFERENCES}

1. R. Steele, Ed., "Fourth International Conference on Plastic Optical Fibers \& Applications," Information Gatekeeper, Boston, MA, USA, Oct. 17-19, 1995.

2. Y. Koike and A. Harmer, Ed., "Third International Conference on Plastic Optical Fibers \& Applications," Yokohama, Japan,
Oct. $26-28,1994$.

3. L. G. Atkinson, D. S. Kindred, and J. B. Zinter, Opt. Photonics News, 5, 28 (1994).

4. Y. Koike, in "Polymers for Lightwave and Integrated Optics," L. A. Hornak, Ed., Marcel Dekker, New York, N.Y., 1992.

5. D. T. Moore, Appl. Opt., 19, 1035 (1980).

6. T. Ishigure, E. Nihei, S. Yamazaki, K. Kobayashi, and Y. Koike, Electro. Lett., 31, 467 (1995).

7. A. Tagaya, Y. Koike, E. Nihei, S. Teramoto, K. Fujii, T. Yamamoto, and K. Sasaki, Appl. Opt., 34, 988 (1995).

8. T. Yamamoto, Y. Mishina, and M. Oda (Mitsubishi Rayon Co., Ltd.), US Patent 4,852,982 (1989).

9. N. Toyoda, Y. Mishina, R. Murata, Y. Uozu, M. Oda, and T. Ishimaru (Mitsubishi Rayon Co., Ltd.), US Patent 5390274 (1995).

10. R. Olshansky and D. B. Keck, Appl. Opt., 15, 483 (1976).

11. G. A. Perry and C. E. Witcher (Peachtree Fiberoptics, Inc.), US Patent 5,235,660 (1993).

12. B. C. Ho, J. H. Chen, W. C. Chen, Y. H. Chang, S. Y. Yang, J. J. Chen, and T. W. Tseng, Polym. J., 27, 310 (1995).

13. W. C. Chen, J. H. Chen, S. Y. Yang, J. Y. Cherng, Y. H. Chang, and B. C. Ho, J. Appl. Polym. Sci., 60, 1379 (1996).

14. W. C. Chen, J. H. Chen, S. Y. Yang, J. J. Chen, Y. H. Chang, B. C. Ho, and T. W. Tseng, "Photonic and Optolectronic Polymers," Vol. 672, S. A. Jenekhe and K. J. Wynne, Ed., ACS Symposium Series, in press.

15. Y. Mishina, Y. Uotsu, and M. Oda (Mitsubishi Rayon Co., Ltd.), JP Patent, 1-189602 (1989).

16. Y. Mishina, Y. Uotsu, and M. Oda (Mitsubishi Rayon Co., Ltd.), JP Patent, 2-16505 (1990).

17. Y. Mishina, R. Murata, Y. Uotsu, and M. Oda (Mitsubishi Rayon Co., Ltd.), JP Patent, 2-33104 (1990).

18. B. T. Liu, W. C. Chen, and J. P. Hsu, Polymer, 40, 1451 (1999)

19. H. A. Lorentz, Wied. Ann. Phys., 9, 641 (1880).

20. L. V. Lorenz, Wied. Ann. Phys., 11, 70 (1880).

21. D. W. Van Krevelen, "Properties of Polymers," 2nd ed, Elsevier, Amsterdam, 1976.

22. R. E. Treybal, "Mass Transfer Operations," McGraw-Hill, New York, N.Y., 1980. 